\title{
GINGIVAL AND PERIODONTAL DISEASES IN CHILDHOOD - A REVIEW
}

\author{
Ramakrishnan Hemalatha1, Venugopal Leelarani², Kanapathy Ramalingam Shakila ${ }^{3}$
}

${ }^{1}$ Reader, Department of Periodontics, Karpaga Vinayaga Institute of Dental Sciences.

${ }^{2}$ Reader, Department of Paedodontics, Karpaga Vinayaga Institute of Dental Sciences.

${ }^{3}$ Reader, Department of Oral Medicine and Radiology, Karpaga Vinayaga Institute of Dental Sciences.

\section{ABSTRACT}

\section{BACKGROUND}

Gingival and Periodontal diseases, besides affecting adults can also be prevalent in children. Though the occurrence of destructive periodontal diseases in children is lesser than in adults, they can develop severe forms of periodontal diseases. This review article gives an insight about various gingival and periodontal diseases and their management in childhood, thereby reinforcing the importance of maintaining a good periodontal health failing which can lead to disturbances in child's routine ranging from mild discomfort as hindrance in feeding to as severe as tooth loss.

\section{KEYWORDS}

Gingival Disease, Periodontal Disease, Children.

HOW TO CITE THIS ARTICLE: Hemalatha R, Leelarani V, Shakila KR. Gingival and periodontal diseases in childhood - a review. J. Evolution Med. Dent. Sci. 2017;6(12):983-986, DOI: 10.14260/Jemds/2017/210

\section{BACKGROUND}

Gingival and periodontal diseases affect children as much as adults. The most common cause of periodontal disease is dental plaque. The gingival disease can be broadly classified into dental plaque induced and non-plaque induced gingival disease. Other than plaque induced gingival disease, periodontal manifestations can also be a mirror reflecting some of the systemic disorders and diseases. Early screening, diagnosis and treatment could help rule out the cause of the disease in initial stage.

\section{Periodontium of Primary Dentition}

The normal gingiva of primary dentition is different from that of adults. The pale pink colour is less marked in children than attached gingiva of adults, because the thin keratinised layer causes the underlying vessels in children more visible. ${ }^{1}$ Depth of gingival sulcus is shallower in primary dentition than in permanent dentition. The gingival width increases with age as children transit from primary to permanent dentition. ${ }^{2}$ Junctional epithelium is thicker in primary dentition than in permanent dentition, which might reduce the permeability of epithelium to bacterial toxins. Radiographically, the lamina dura is prominent in primary dentition with a wider periodontal ligament space than the permanent dentition.

\section{Gingival Disease \\ Classification \\ Gingivitis Associated with Dental Plaque \\ A. Chronic Gingivitis. \\ B. Eruption Gingivitis. \\ C. Gingival disease modified by Systemic Factors.}

Financial or Other, Competing Interest: None.

Submission 31-12-2016, Peer Review 24-01-2017,

Acceptance 30-01-2017, Published 09-02-2017.

Corresponding Author:

Dr. Ramakrishnan Hemalatha,

W/o. Dr. R. Arun Kumar,

Plot. No. 93, Mahalakshmi Nagar,

Nandivaram, Guduvanchery, Kancheepuram-603202.

E-mail: hemaarun21@gmail.com

DOI: $10.14260 /$ jemds $/ 2017 / 210$

\section{(c) $(1) \$$}

1. Associated with endocrine system.

- Puberty gingivitis.

- Diabetes mellitus associated gingivitis.

2. Associated with blood dyscrasias.

- Leukaemia associated gingivitis.

3. Associated with nutritional deficiency.

- Ascorbic acid deficiency gingivitis.

D. Modified by Medications.

4. Drug induced gingival enlargement.

Non-Plaque Induced Gingivitis

A. Gingival disease of viral origin.

- Primary herpetic gingivostomatitis.

B. Gingival disease of fungal origin.

- Acute candidiasis.

C. Gingival disease of bacterial origin.

- Acute necrotising ulcerative gingivitis.

D. Congenital gingival anomalies.

- Congenital epulis.

E. Traumatic gingival lesions.

- Factitious gingivitis, accidental, iatrogenic.

\section{Chronic Gingivitis}

The primary aetiology being plaque, gingivitis is regarded as the most common periodontal disease in children. ${ }^{3}$ Accumulation of food debris, plaque and microorganisms initiates inflammation leading to gingivitis. If it is not taken care of in the initial stage, it will progress to periodontitis. Inflammation is limited to marginal gingival without bone loss or connective tissue attachment loss. Bleeding gums is a significant clinical feature. Calculus deposits are rarely seen in infants, but may increase with age.

\section{Eruption Gingivitis}

Gingival inflammation occurs around an erupting permanent tooth. The child may have difficulty and discomfort in brushing, making the areas more vulnerable for plaque accumulation. Eruption gingivitis is usually mild, needs no treatment but improved oral hygiene. 


\section{Gingival Disease Modified by Systemic Factors Gingivitis Associated with Diabetes Mellitus}

Type I diabetes mellitus is the juvenile onset type affecting young people. Alteration in immune function, impaired glucose uptake by liver, premature apoptosis is abnormalities related to diabetes mellitus. ${ }^{4}$ In children with poor metabolic control, gingival inflammation and periodontitis are common. ${ }^{5}$ In severe cases, premature tooth loss and impaired immune response to oral flora is seen. Treatment includes disease prevention and control of diabetes. ${ }^{6}$

\section{Puberty Gingivitis}

Usually seen around 11 - 14 years of age. Hormonal changes during puberty may exaggerated vascular and inflammatory response to dental plaque. Gingival enlargement; bleeding and inflammation are common findings. This condition usually subsides after puberty.

\section{Gingivitis Associated with Haematological Disorders}

Leukaemia is the most common type of cancer. Leukaemic patients present bleeding and diathesis, petechiae oral ecchymosis, gingival haemorrhage and progressive gingival enlargement.7,8 They have lowered tissue resistance to infections due to reduced WBC count. So, vigorous plaque control measures should be implicated before commencing cytotoxic treatment and during medical treatment.

Gingivitis associated with vitamin $\mathrm{C}$ deficiency can cause spongy and oedematous gingiva with spontaneous bleeding and impaired wound healing. Treatment includes correction of underlying deficiency along with plaque control. Chronic deficiency of iron, vitamin B group and folic acid also predispose to glossitis and gingivitis especially in children.

In plaque induced gingival enlargement, there is localised or generalised enlargement of interdental papilla and gingival margin. Clinically, gingiva appears pale and fibrotic to red and friable. In addition to strict plaque control measures, gingivectomy or gingivoplasty may be indicated.

Drug induced gingival enlargement is a side effect of drugs like phenytoin, antiepileptic and cyclosporine immunosuppressant. Gingival enlargement is the most significant oral finding. ${ }^{9}$ Enlargement is mulberry shaped, pink, firm and stippled. In patients with pre-existing gingivitis, enlarged tissues show signs of gingivitis. Meticulous oral hygiene measures and scaling must be implemented.

\section{Herpetic Gingivostomatitis}

Also known as acute herpetic gingivostomatitis. It affects both gingiva and other parts of oral mucous membrane. It is caused by Herpes Simplex Virus (HSV) type I, most commonly affecting children between 2 - 5 years of age. ${ }^{10}$ Infection usually follows episodes of childhood fever such as malaria, measles and chicken pox. Clinical features include fever, headache, malaise, mild dysphagia and cervical lymphadenopathy. Gingivitis is the most significant feature with swollen, erythematous and friable gums. The condition is self-limiting and management includes bed rest and plenty of fluid intake. Gentle debridement of the lesion is done to maintain good oral hygiene. Analgesics are given to relieve pain and antibiotics to prevent superimposed bacterial infections. A mild topical anaesthetic gel will reduce irritability.

Gingivitis caused by candidia albicans is less common in children and is rarely associated with healthy child. It may occur following a course of antibiotics or in neonates infection contracted during passage through vagina. Clinically, the condition is seen as furry white patches, removal of which leaves behind a bleeding surface. Drug of choice in older children is clotrimazole, while in infants a suspension of $1 \mathrm{~mL}(1,00,000 \mathrm{U})$ of nystatin four times a day is given.

\section{ANUG}

This is an acute bacterial infection of gingiva. The lesion starts at the interdental papilla, spreads along the gingival margin if untreated. It is an anaerobic infection caused by fusiform bacteria, spirochetes and other gram negative anaerobic microorganisms. The predisposing factors are malnutrition, stress and lack of sleep. It is common in young children in less developed countries. Onset of the disease is rapid and painful. The clinical features are 'punched out' ulceration and necrosis of interdental papilla and marginal gingiva covered by yellowish grey pseudomembranous slough. Treatment includes meticulous hygiene measures, plaque control, mouth rinse $\left(0.5 \% \mathrm{H}_{2} \mathrm{O}_{2}\right.$ - removal of necrotic tissue, $0.2 \%$ Chlorhexidine - prevents plaque formation). ${ }^{11}$

Traumatic lesions can be due to chemical, thermal injury or (tooth brush abrasion). This condition presents as a painful ulceration surrounded by erythematous halo. The child has to be taught to follow the right brushing technique.

Congenital epulis, a rare tumour along the alveolar ridge in new-borns presents as a smooth, well-defined erythematous mass arising from the gum pads. Larger lesions have to be resected as they can hinder feeding of the baby. The unerupted teeth are usually unaffected.12

\section{Periodontal Disease}

1. Chronic periodontitis.

2. Aggressive periodontitis.

3. Periodontitis as a manifestation of systemic disease.

4. Necrotising ulcerative periodontitis.

\section{Aggressive Periodontitis}

Aggressive periodontitis can be localised or generalised.

Localised aggressive periodontitis occurs in children, adolescents with non-contributory medical history and is characterised by severe alveolar bone loss around permanent teeth, ${ }^{13}$ usually localised aggressive periodontitis is restricted to permanent first molars and incisors. Interproximal attachment loss is seen in at least two permanent first molars and incisors with attachment loss in not more than two teeth other than first molars and incisors. The primary feature of aggressive periodontitis includes a history of rapid attachment and bone loss with familial aggregation. Secondary features include phagocyctic abnormalities and a hyperresponsive macrophage phenotype. ${ }^{14}$

A number of functional defects reported in neutrophils from patients with localised aggressive periodontitis includes chemotaxis defects, ${ }^{15,16}$ defects in phagocytosis, ${ }^{17,18}$ bactericidal activity ${ }^{19}$ and superoxide production. 


\section{Generalised Aggressive Periodontitis}

Exhibits generalised interproximal attachment loss including at least three teeth that are not first molars and incisors. This disease usually has a circumpubertal onset in young individuals. There is marked periodontal inflammation with heavy plaque and calculus accumulation. Increased number of non-motile, facultative anaerobic, gram negative rods including Porphyromonas gingivalis is present.20 In generalised aggressive periodontitis, neutrophils exhibit suppressed chemotaxis as in localised aggressive periodontitis ${ }^{21}$ suggesting a relationship between two variants of aggressive periodontitis. ${ }^{22}$ Early diagnosis and treatment can give successful results.

Use of antibiotics in conjunction with surgical or nonsurgical root debridement appears to be quite effective for treating localised aggressive periodontitis, whereas generalised aggressive periodontitis does not always respond well to conventional mechanical therapy or to antibiotics commonly used to treat periodontitis.

\section{Chronic Periodontitis}

Though chronic periodontitis is most prevalent in adults, it can occur in children and adolescents. It can be localised (< $30 \%$ of dentition) or generalised ( $>30 \%$ of dentition). It is characterised by slow-to-moderate rate of disease progression including periods of quiescence. Severity of disease can be mild ( $1-2 \mathrm{~mm}$ clinical attachment loss), moderate (3 - $4 \mathrm{~mm}$ clinical attachment loss), severe (>5 mm clinical attachment loss).

\section{Periodontitis Associated with Systemic Disease}

Can occur in children and adolescents which include PapillonLefevre syndrome, cyclic neutropenia, agranulocytosis, Down syndrome, hypophosphatasia and leucocyte adhesion deficiency. Defects in neutrophil and immune cell function may play a role in increased susceptibility to periodontitis and other infection.

\section{Necrotising Periodontal Disease}

The most characteristic clinical findings in necrotising periodontal disease is the presence of interproximal necrosis and ulceration with rapid onset of gingival pain. Patient may be febrile. High levels of P. intermedia and spirochetes are present. Some of the common predisposing factors are malnutrition, viral infections (including HIV), emotional stress, lack of sleep and other systemic diseases. Treatment includes mechanical debridement, oral hygiene instruction and followup. ${ }^{23}$

\section{CONCLUSION}

Though prevalence of destructive periodontal diseases in children is lesser than in adults, still they may develop severe forms of periodontitis. In some conditions, the destructive disease may be a manifestation of known underlying systemic disease, while in others the underlying cause is unknown. The array of gingival and periodontal disease can range from trivial forms of gingivitis due to plaque and calculus (local factors) to aggressive forms causing tooth loss. Early diagnosis with vigilant and prompt treatment can curb severe forms of disease. So, screening for periodontal problems in children should be mandatory as part of routine dental visits.

\section{REFERENCES}

[1] Maynard JG, Ochsenbein C. Mucogingival problems, prevalence and therapy in children. J Periodontol 1975;46(9):543-52.

[2] Andlin-Sobocki A, Bodin L. Dimensional alterations of the gingiva related to changes of facial/lingual tooth position in permanent anterior teeth of children. A 2year longitudinal study. J Clin Periodontol 1993;20(3):219-24.

[3] Oh TJ, Eber R, Wang HL. Periodontal disease in the child and adolescent. Journal of Clinical Periodontology 2002;29(5):400-10.

[4] Acikgoz G, Devrim I, Ozdamar S. Comparison of keratinocyte proliferation in diabetic and non-diabetic inflamed gingiva. Journal of Periodontology 2004;75(7):989-94.

[5] Pinson M, Hoffman WH, Garnick JJ, et al. Periodontal disease and type diabetes mellitus in children and adolescents. J Clin Periodontol 1995;22(2):118-23.

[6] Lalla E, Cheng B, Lal S, et al. Periodontal changes in children and adolescents with diabetes: a case-control study. Diabetes Care 2006;29(2):295-9.

[7] Weckx LL, Hidal LB, Marcucci G. Oral manifestations of leukemia. Ear Nose and Throat 1990;69(5):341-2, 345-6.

[8] Genc A, Atalay T, Gedikoglu G, et al. Leukemic children: clinical and histopathological gingival lesions. Journal of Clinical Pediatr Dentistry 1998;22(3):253-6.

[9] Robbins MR. Dental management of special needs patients who have epilepsy. Dental Clinics of North America 2009;53(2):295-309.

[10] Sapp JP, Eversole LR, Wysocki GP. Contemporary oral and maxillofacial pathology. $2^{\text {nd }}$ edn. St. Louis, Mosby (Elsevier) 2004.

[11] Koch G, Poulsen S. Periodontal conditions. 2nd edn. Pediatric dentistry a clinical approach. WileyBlackwell 2009:p180.

[12] Koch BL, Myer C, Egelhoff JC. Congenital epulis. AJNR 1997;18:739-41.

[13] Baer PN. The case for periodontosis as a clinical entity. J Periodontol 1971;42(8):516-20.

[14] Armitage GC. Development of a classification system for periodontal diseases and conditions. Ann Periodontol 1999;4:1-6.

[15] Genco RJ, Dyke VTE, Levine MJ, et al. 1985 Kreshover lecture. Molecular factors influencing neutrophil defects in periodontal disease. J Dent Res 1986;65(12):1379-91.

[16] Dyke VTE, Hoop GA. Neutrophil function and oral disease. Crit Rev Oral Biol Med 1990;1(2):117-33.

[17] Dyke VTE, Zinney W, Winkel K, et al. Neutrophil function in localized juvenile periodontitis. Phagocytosis, superoxide production and specific granule release. J Periodontol 1986;57(11):703-8.

[18] Cogen RB, Roseman JM, Al-Joburi W, et al. Host factors in juvenile periodontitis. J Dent Res 1986;65(3):394-9.

[19] Kalmar JR, Arnold RR, Dyke VTE. Direct interaction of actinobacillus actinomycetemcomitans with normal and defective (LJP) neutrophils. J Periodont Res 1987;22(3):179-81. 
[20] Slots J. Importance of black-pigmented bacteroides in human periodontal disease. In: Genco RJ, Mergenhagen SE, (eds). Host-Parasite interactions in periodontal diseases. Washington, DC: American Society for Microbiology 1982:27-45.

[21] Dyke VTE, Levine MJ, Genco RJ. Periodontal diseases and neutrophil abnormalities. In: Genco RJ, Mergenhagen SE, (eds). Host-Parasite interactions in periodontal diseases. Washington, DC: American Society for Microbiology 1982:235-45.
[22] Dyke VTE, Wilson-Burrows C, Offenbacher S, et al. Association of an abnormality of neutrophil chemotaxis in human periodontal disease with a cell surface protein. Infect Immun 1987;55(9):2262-7.

[23] Enwonwu CO. Epidemiological and biochemical studies of necrotizing ulcerative gingivitis and noma (cancrum oris) in Nigerian children. Arch Oral Biol 1972;17(9):1357-71. 